\title{
Production of methanol and oxymethylene on an industrial scale
}

Torsten Buddenberg, Co-Authors: Dr. Christian Bergins, both Mitsubishi Hitachi Power Systems Europe $\mathrm{GmbH}$, Wolfgang Maus, Continental Emitec $\mathrm{GmbH}$, K. C. Tran, Carbon Recycling International, Iceland

Zum Zeitpunkt der Drucklegung lagen diese Unterlagen noch nicht vor. Wir bitten um Verständnis. 